\title{
Distribution Pattern of ER \& PR Immunoexpression in Endometrial Biopsies of DUB and Infertile Patients from A Tertiary Care Centre
}

\author{
Brijesh Thakur, Sanjay Kaushik*, Sakshi Garg, Sanjeev Kishore \\ Shri Guru Ram Rai Institute of Medical \& Health Sciences, Dehradun, India
}

\begin{abstract}
Objective: To study endometrial estrogen and progesterone immunoexpression in different phases of menstrual cycle, in cases of dysfunctional uterine bleeding and infertility. A comparative analysis was also done for calculating ER \& PR expression, between two methods: quick score and percentage of immunopositive cells.

Methods: Endometrial biopsies from 107 clinically diagnosed DUB cases and 23 infertile patients were included in the study. Tissue sections were analyzed for different phases of menstrual cycle and immunoexpression of ER \& PR receptors was calculated in glandular epithelium and stromal cells, using percentage of positively stained cells and Quick score method. Endometrial sections of hysterectomy specimens of uterovaginal prolapse cases were used as control sections.
\end{abstract}

Result: In the present analysis, secretory endometrium was the commonest finding histopathologically.Mean total ER \& PR expression in cases of DUB was statistically higher in the proliferative phase using both methods $(\mathrm{p}<0.05)$. Mean total ER expression difference in both the phases of infertility cases was not statistically significant by the observation of percentage of positively stained cells. However, quick score revealed significant difference between both receptor expression in infertile patients.

Conclusion: The percentage of positive cells for ER and PR expression plays a more determinant role in studying ER and PR expression in cases of DUB and infertility as compared to quick score.

Keywords: Endometrium, DUB, Infertility, Quick score

\section{Introduction}

Infertility and dysfunctional uterine bleeding (DUB) together form the most common presenting complaint in obstetrics and gynaecology OPDs. Infertility is more of a social problem, whereas DUB is one of the most bothering complaints for any female, both mentally and physically. A common point, shared by these two conditions is that their prompt diagnosis and timely treatment can significantly raise the living standards of the patient. Dysfunctional uterine bleeding is defined as bleeding not associated with an organic cause in women of child bearing age. ${ }^{[1]}$ Anovulatory cycles play an aetiological role in about $90 \%$ of cases of DUB. Infertility is termed when a couple fails to achieve pregnancy after 1 year of unprotected and regular intercourse. ${ }^{[2]}$ Changing lifestyle of people has gradually increased the incidence of infertility.

The uterine endometrium is exquisitely sensitive to estrogens and progesterone which play important role in sexual development and reproduction. The estrogen receptor (ER) and progesterone receptor (PR) expression and distribution pattern have been demonstrated to play an important role in normal endometrial functioning. Immunohistochemistry has essential clinical implications since it supports the role of hormone receptors in the etiopathogenesis of DUB and also it could start a new field in the hormonal therapy. ${ }^{[3,4]}$

The present study was aimed to analyze endometrial estrogen and progesterone immunoexpression in different phases of menstrual cycle, in cases of dysfunctional uterine bleeding and infertility. A comparison was also done for calculating ER \& PR expression, between two methods: quick score and percentage of immunopositive cells.

\section{Material and Methods}

Total 150 clinically diagnosed cases of DUB and infertility over a period of two years were included in the present study. Out of them, 20 cases had fibroids as the cause of bleeding and were excluded from the study. Out of remaining 130 cases, 107 were DUB cases and 23 were infertility cases (20 cases - primary infertility and 3 cases - secondary infertility). All DUB cases presented with menorrhagia. Ten hysterectomy specimens of uterovaginal prolapse in the reproductive age group (21- 
$50 \mathrm{yrs})$ were taken as controls. Control section was run with each set of 13 cases.

Tissue samples were collected as endometrial biopsies (in premenstrual phase) and fixed in 10\% formalin. Sections of 3-5 micron were made, stained with haematoxylin \& eosin and were reported. For IHC, sections of 2-4 micron from the paraffin embedded blocks were taken on Poly-L lysine coated slides and subjected to ER \& PR staining by indirect method of immunohistochemistry using rabbit monoclonal antibodies of Cell Marque; PR clone Y85 at dilution 1:100 and ER clone EP1 at dilution 1:150.

The expression of ER \& PR was assessed in the endometrial stroma and glands using semiquantitative method, Quick score of nuclear reaction. The percentage of positively stained cells and intensity of nuclear staining was recorded and final quick score was calculated by adding the obtained grading scores of percentage positive cells and the intensity.(Table 1)

Subsequently the data was tabulated in mean values of to ER \& PR expression and analysed. Statistical analysis was done using IBM SPSS software version 20. Student ' $t$ ' test was used in the present study to find out the significance $(\mathrm{p}<0.05$ taken as significant $)$.

\section{Results}

In the present study, major proportion of subjects was young adults in age group of 21-40 years. Amongst DUB cases, maximum cases (50 cases, $46.73 \%$ ), were seen in the age group 31-40 yrs and minimum cases ( 4 cases, 3.74\%) were seen in the age group 21-30 yrs. 18 cases of infertility (78.26\%) were seen in the 20-30 yrs of age group and rest of the 5 cases (13.04\%) were in the age group of 31-40 yrs.

According to histopathology, $60 \%$ of control cases were in proliferative phase and $40 \%$ were in secretory phase. In total 107 DUB cases, the maximum cases (36.4\%) were in secretory phase, followed by irregular shedding endometrium (26.2\%), simple hyperplasia without atypia (18.7\%) and proliferative phase (18.7\%). Out of total 23 infertility cases, the maximum cases $(47.82 \%)$ were in secretory phase, followed by proliferative and irregular shedding endometrium equally ( $21.73 \%$ each). Simple hyperplasia without atypia constituted $8.69 \%$ of infertile cases.

In control cases, taking into account the percentage of positive cells, total mean ER \& PR expression was significantly higher in the proliferative phase, as compared to secretory phase (for ER $\mathrm{p}=<0.0001$, for $\mathrm{PR} p=<0.001$ ). Mean total ER expression was $90.84 \%$ in proliferative phase while $39.13 \%$ in secretory phase. Mean total PR expression was $84.0 \%$ in proliferative phase while $63.13 \%$ in secretory phase in control sections. Mean total ER expression in cases of DUB was high in the proliferative phase $(86.50 \%)$ while in the secretory phase was $59.68 \%$. Mean total PR expression was also found high in the proliferative phase $(79.70 \%)$ and $53.71 \%$ was in the secretory phase. These differences in ER \& PR expression were found statistically significant in both phases of DUB cases. Mean total ER expression difference in both the phases of infertility cases $(82.90 \%$ \& $66.14 \%)$ was not statistically significant which denotes that the ER expression was consistently raised during the secretory phase. However, mean total PR expression was statistically significant in infertility cases in both phases $(82.4 \%$ \& $57.14 \%)$.(TABLE 2$)$

Taking staining intensity into account, mean ER and PR staining intensity was higher in proliferative phase as compared to secretory phase but not, statistically significant in both phases of control and infertile cases. In DUB patients, ER and PR staining intensity results followed the same pattern as control cases except the fact that mean glandular PR expression intensity difference was statistically significant in between both phases.(Table 3)

In control cases, distribution of quick score of mean total ER \& PR expression was high in proliferative phase with statistically significant difference $(\mathrm{p}=<0.05)$ as compared to secretory phase. In DUB group, mean total ER and PR quick score was higher in proliferative phase being statistically very significant $(p=<0.0001)$.In infertile patients, mean total ER expression score was significantly higher in the proliferative phase while on the other hand the mean total PR quick score was higher $(\mathrm{p}=<0.05)$ in the secretory phase.(Table 4)

Table 1: Grading of ER \& PR expression according to positivity of cells and intensity of nuclear staining.

\begin{tabular}{|c|c|c|c|}
\hline \multicolumn{2}{|c|}{ Percentage of stained cells } & \multicolumn{2}{c|}{ Intensity of nuclear staining } \\
\hline Grade 0 & none stained & Grade 0 & negative \\
\hline Grade 1 & $<1 \%$ of cells stained & Grade 1 & weak reaction \\
\hline Grade 2 & $110 \%$ of cells stained & Grade 2 & moderate reaction \\
\hline Grade 3 & $1133 \%$ of cells stained & Grade 3 & intense reaction \\
\hline Grade 4 & $3466 \%$ of cells stained & & \\
\hline
\end{tabular}


Table 2: Distribution \& comparison of ER \& PR expression (\% of positive cells) in Control, DUB \& Infertility Cases.

\begin{tabular}{|c|c|c|c|c|c|c|}
\hline & \multicolumn{2}{|c|}{ CONTROL } & \multicolumn{2}{|c|}{ DUB } & \multicolumn{2}{|c|}{ INFERTILITY } \\
\hline & PP & SP & PP & SP & PP & SP \\
\hline Mean total ER expression & 90.84 & 39.13 & 86.50 & 59.68 & 82.90 & 66.14 \\
\hline P Value & \multicolumn{2}{|c|}{$<0.0001$} & \multicolumn{2}{|c|}{$<0.0001$} & \multicolumn{2}{|c|}{$>0.05$} \\
\hline Mean total PR expression & 84.00 & 63.13 & 79.70 & 53.71 & 82.40 & 57.14 \\
\hline P Value & \multicolumn{2}{|c|}{$<0.001$} & \multicolumn{2}{|c|}{$<0.0001$} & \multicolumn{2}{|c|}{$<0.05$} \\
\hline
\end{tabular}

PP- PROLIFERATIVE PHASE SP-SECRETORY PHASE

Table 3: Distribution \& comparison of ER \& PR expression (Intensity of nuclear staining) in Control, DUB \& Infertility Cases.

\begin{tabular}{|c|c|c|c|c|c|c|}
\hline & \multicolumn{2}{|c|}{ CONTROL } & \multicolumn{2}{|c|}{ DUB } & \multicolumn{2}{|c|}{ INFERTILITY } \\
\hline & PP & SP & PP & SP & PP & SP \\
\hline Mean total ER expression & 1.67 & 1.00 & 1.92 & 1.56 & 1.60 & 1.00 \\
\hline P Value & \multicolumn{2}{|c|}{$>0.05$} & \multicolumn{2}{|c|}{$>0.05$} & \multicolumn{2}{|c|}{$>0.05$} \\
\hline Mean total PR expression & 1.42 & 1.50 & 1.84 & 1.40 & 1.20 & 1.18 \\
\hline P Value & \multicolumn{2}{|c|}{$>0.05$} & \multicolumn{2}{|c|}{$>0.05$} & \multicolumn{2}{|c|}{$>0.05$} \\
\hline
\end{tabular}

PP-PROLIFERATIVE PHASE SP-SECRETORY PHASE

Table 4: Distribution \& comparison of ER \& PR expression (QUICK SCORE) in Control, DUB \& Infertility Cases.

\begin{tabular}{|c|c|c|c|c|c|c|}
\hline & \multicolumn{2}{|c|}{ CONTROL } & \multicolumn{2}{|c|}{ DUB } & \multicolumn{2}{|c|}{ INFERTILITY } \\
\hline & PP & SP & PP & SP & PP & SP \\
\hline Mean total ER expression & 6.67 & 5.00 & 6.86 & 5.94 & 6.60 & 4.07 \\
\hline P Value & \multicolumn{2}{|c|}{$<0.05$} & \multicolumn{2}{|c|}{$<0.0001$} & \multicolumn{2}{|c|}{$<0.05$} \\
\hline Mean total PR expression & 6.42 & 5.63 & 6.64 & 5.50 & 5.21 & 6.03 \\
\hline $\mathrm{P}$ Value & \multicolumn{2}{|c|}{$<0.05$} & \multicolumn{2}{|c|}{$<0.001$} & \multicolumn{2}{|c|}{$<0.05$} \\
\hline
\end{tabular}

PP- PROLIFERATIVE PHASE SP-SECRETORY PHASE

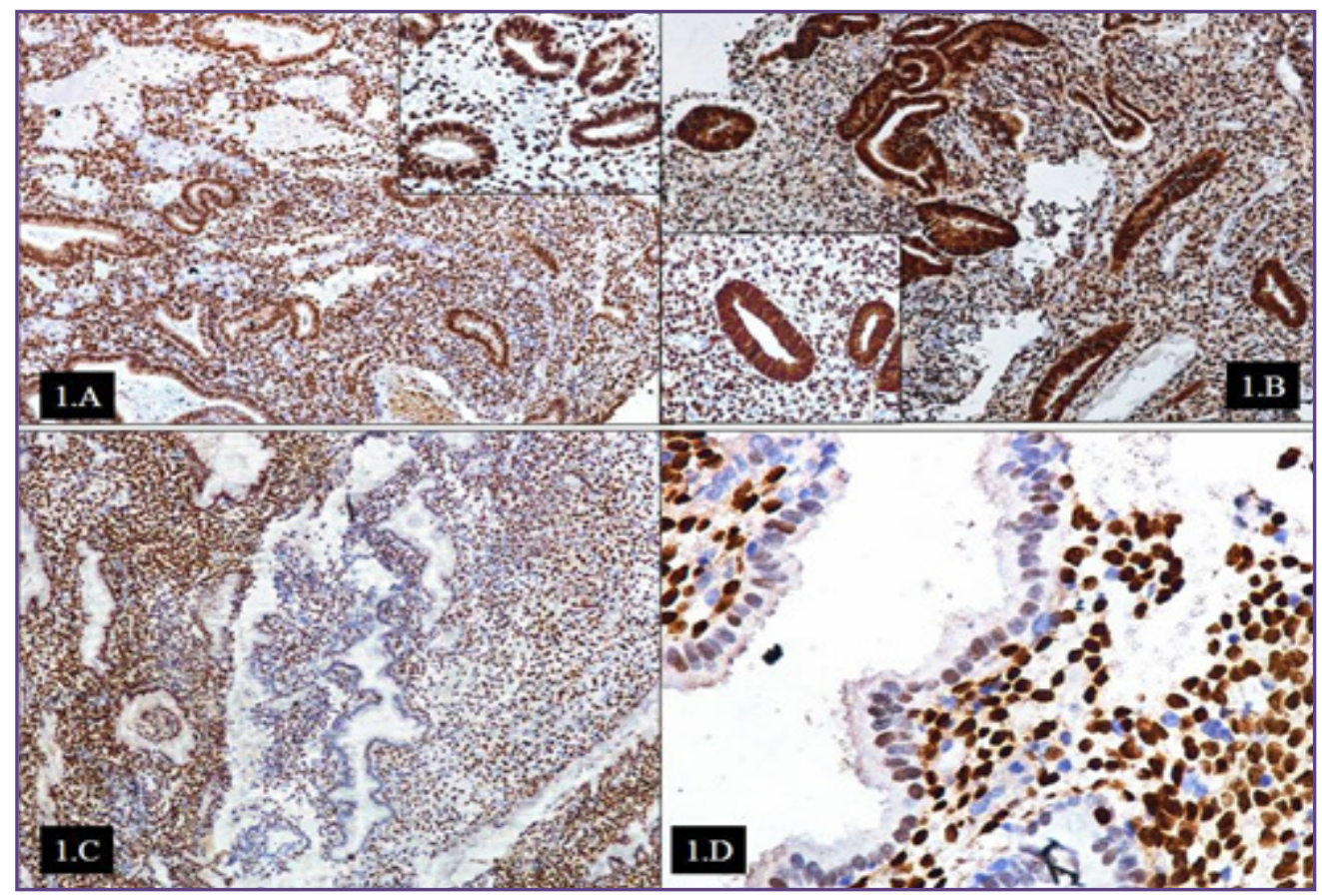

Fig. 1: A. IHC Showing ER expression in endometrial glands and stroma in proliferative phase (100x). Inset showingER expression in endometrial glands in proliferative phase (400x).; 1.B. IHC Showing PR expression, in endometrial glands and stroma in proliferative phase(100x). Inset showingPR expression in endometrial glands(400x).; 1.C. IHC showing ER expression in endometrial glands and stroma in secretory phase (100x).; 1.D. IHC showing PR expression in endometrial glands and stroma in secretory phase (400x). 


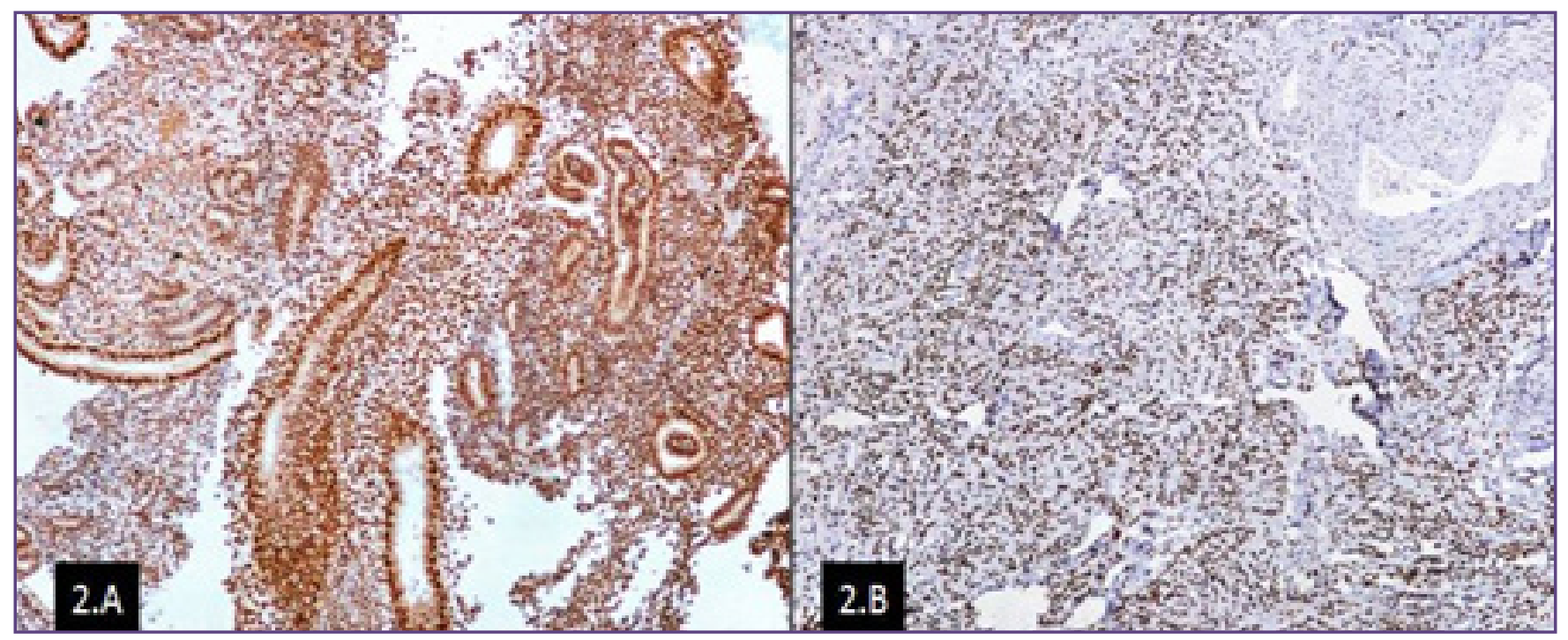

Fig. 2: A. IHC showing ER expression in endometrial glands and stroma exhibiting simple hyperplasia without atypia (100x).; 2.B. IHC showing positive ER expression in myometrium (black arrow)and negative ER expression in myometrial vessel (red arrow) (100x).

\section{Discussion}

Despite the fact that the endometrium is a prime target organ for actions of estrogen and progesterone hormones and hormonal imbalance underlies many gynecological disorders, the clinical significance of endometrial ER and PR expression is underrated in literature. Functional disturbances with hormonal dysfunction may differ, but subtle morphological changes may not differ much. Study of endometrium for ER and PR expression may serve this purpose because the endometrial study has a distinct advantage over biochemical determination of hormones, because the actual effects of these hormones can be studied in the target tissue.

In our study, out of the 107 cases of DUB, a major proportion of cases (50 cases, $46.73 \%$ ) were from the age group 31-40 years. A comparable distribution of patients in different age groups has been reported by Gleeson et al, Chakraborty et al and Chakravarthy et al. ${ }^{[3,5,6]} \mathrm{A}$ few studies by Dhadhania et al and Gupta et al have however reported major number of patients from the age group of 41-50 yrs age group..$^{[7,8]}$ The literature reflects the sociocultural differences between Indian and western communities, as marriages occur earlier in India than western world. Maximum cases of infertility (18 cases, $78.26 \%$ ) were in the age group of 21-30 years. Emokpae et al, also observed the mean age of their cases to be in the range of 21-30 yrs. ${ }^{[9]}$

Abdullah et al and Shanthala et al reported secretory phase as the most common pattern on histopathology in DUB cases as found in our study. ${ }^{[10,11]}$ Emokpae et al who also reported secretory endometrium as the most common pattern similar to the present study. ${ }^{[9]}$ In our study we studied ER and PR expression in endometrial glands and stroma separately as well as a total expression. We found that the maximum ER and PR expression was noted in the glandular epithelium, except for PR expression in the secretory phase which was more in the stromal cells than glandular epithelial cells, a finding supported by Press et al. ${ }^{[12]}$ Expression of these receptors was observed to be absent from vascular smooth muscle and endothelial cells in both endometrium and myometrium in the present study; also reported by Chakraborty et al, Press et al and Snijders et al. ${ }^{[5,12,13]}$ It was also noted during our study that the method of endometrial sampling did not affect the demonstration of ER and PR expression, a finding corroborated by study of Garcia et al. ${ }^{[14]}$

Antigen retrieval for ER is considered to be difficult as stated by Shet $\mathrm{T}$ et al, ${ }^{[15]}$ but we did not find any significant discrepancy in the staining intensity for ER and PR. Although, staining for PR was more crisp and brighter than that for ER in most of our cases, which can be easily explained by the quantitative data from steroid-binding assays indicating that the content of PR in femtomoles per milligram cytosol protein is greater than ER. Press et al and Garcia et al also found that the PR staining was brighter and showed stronger intensity as compared to ER staining. ${ }^{[12,14]}$

In this study, we examined the whole endometrial section for quantification of positively stained cells and results were expressed as percentage positivity, staining intensity and Quick score. Snijders et al and Lessey et al also used semiquantitative scoring methods in their studies of ER 
and PR expression, which accounted both intensity and percentage of stained cells. ${ }^{[13,16]}$ However, we found that there was subjective and inter observer variation in assigning a score for intensity of staining and therefore did not correlate well with the percentage positivity. Also in some cases insufficient antigen retrieval resulted in weak staining.

In the present study, the mean percentage positive ER and PR expression in control cases showed cyclical variation also reported by Press et al and Snijders et al. ${ }^{[12,13]}$ Also in DUB cases, the trend of mean percentage positive ER \& PR expression was high during the proliferative phase probably resulted from the increased local estradiol concentration. Similar findings were observed by Chakraborty et al, Shet $\mathrm{T}$ et al, and Gleeson et al, stating that the trend of ER and PR expression in DUB cases was consistent with the findings in normal control endometrium. ${ }^{[3,15,17]}$ Mean staining intensity of ER \& PR expression in DUB cases was found higher in the proliferative phase and low in the secretory phase. However, this pattern did not correspond statistically with the mean percentage positive cell pattern of the receptor's expression in DUB cases, which may be due to the interobserver variation in assigning the staining score.

In our present study on comparing the mean Quick scores of ER and PR expression in DUB cases, it was noted that the score was corresponding with the percentage positivity of ER and PR expression.The ER staining intensity did not match with the percentage positive expression of ER in both phases, which can be possibly due to the local increase of estradiol in DUB cases during the secretory phase. So a poor concordance among observers may be suggested in assigning score to intensity parameter except in very strongly positive or completely negative sections. In our study,quick score corresponded well with the percentage positive ER and PR expression in DUB cases, similarlyfound by Wells et al. ${ }^{[18]}$

There was an increasing trend of ER and PR expression in proliferative phase and decreasing trend in secretory phase in infertile patients. The difference in ER expression in both the phases was however not statistically significant ( $p>0.05$ ), which denotes that the ER expression was raised during the secretory phase. The mean percentage positivity of PR expression was slightly raised in the stroma in the secretary phase attributed to the increased PR expression in the stroma during the secretary phase. Margarit et al also found raised ER expression in infertile secretory endometrium; also observed a little different finding stating that the intensity of both ER and PR expression intensity was increased in infertile ovulatory endometrium in secretory phase. ${ }^{[19]}$

Our study suggests that mean Quick score for ER expression in glandular, stromal and in total was higher in proliferative phase in infertility cases while the mean total PR expression Quick score was higher in the secretory phase. However, Margarit et al and Papanikalaou et al in their study of 'infertile endometrium' observed increased expression of both receptors in the secretory phase. ${ }^{[19,20]}$

\section{Conclusion}

In conclusion, secretory phase is the appropriate phase for obtaining endometrial biopsy as overlap of ER and PR expression may appear in the proliferative phase. The percentage positive cells for ER and PR expression plays a determinant role in studying ER and PR expression in cases of DUB and infertility. The inter-observer variability while reporting mean staining intensity limits its utilisation. Since Quick score has staining intensity as one of its component we found that the percentage positivity of cells is a more reliable indicator during reporting.

To the best of our knowledge we have not come across any study which has compared percentage positivity of cells, staining intensity and Quick score, all these parameters together, in both phases of menstrual cycle. However, a few studies have reported the singleton use of these parameters in cases of DUB and infertility.

\section{References}

1. Rosai J. Female reproductive system. Uterus-corpus. In: Rosai and Ackerman's Surgical Pathology. Vol 2. 9thed. New Delhi: Elsevier; 2004. p. 1569-635.

2. Dallenbach-Hellweg G. The Endometrium of Infertility. A Review. Pathol Res Pract 1984;178(6):527-37.

3. Chakraborty S, Khurana N, Sharma JB, Chaturvedi KU. Endometrial hormone receptors in women with dysfunctional uterine bleeding. Arch GynecolObstet 2005;272(1):17-22.

4. Mylonas I, Jeschke U, Shabani N, Kuhn C, Balle A, Kriegel S, et al. Immunohistochemical analysis of estrogen receptor alpha, estrogen receptor beta and progesterone receptor in normal human endometrium. ActaHistochem 2004;106(3):245-52.

5. Chakravarthy VK, Nag U, Rangarao D, Anusha AM. Estrogen and progesterone receptors in dysfunctional uterine bleeding. IOSR-JDMS 2013;4(3):73-6.

6. Gleeson N, Jordan M, Sheppard B, Bonnar J. Cyclical variation in endometrial oestrogen and progesterone receptors in women with normal menstruation and dysfunctional uterine bleeding. Eur J ObstetGynecolReprodBiol 1993;48(3):207-14.

7. Dadhania B, Dhruva G, Agravat A, Pujara K. Histopathological study of endometrium in dysfunctional uterine bleeding. J Int Med Res 2013;2(1):20-4. 
8. Gupta A, Rathore AM, Manaktala U, Rudingwa P. Evaluation and histopathological correlation of abnormal uterine bleeding in perimenopausal women. IJBAR 2013;4(8):509-13.

9. Emokpae MA, Uadia PO, Mohammed AZ. Hormonal evaluation and endometrial biopsy in infertile women in Kano, Northern Nigeria: Acomparative study. Ann Afr Med 2005;4(3):99-103.

10. Abdullah LS, Bondagji NS. Histopathological pattern of endometrial sampling performed for abnormal uterine bleeding. Bahrain Med Bull 2011;33(4):1-6.

11. Shet T, Agrawal A, Nadkarni M, Palkar M, Havaldar R, Paramar V, et al. Hormone receptors over the last 8 years in a cancer referral center in India: what was and what is? Indian J PatholMicrobiol 2009;52(2):171-4.

12. Press MF, Udove JA, Greene GL. Progesterone receptor distribution in the human endometrium. Analysis using monoclonal antibodies to the human progesterone receptor. Am J Pathol 1988;131(1):112-24.

13. Snijders MP, de Goeij AF, Debets-TeBaerts MJ, Rousch MJ, Koudstaal J, Bosman FT. Immunocytochemical analysis of oestrogen receptors and progesterone receptors in the human uterus throughout the menstrual cycle and after the menopause. J ReprodFertil 1992;94(2):363-71.

14. Garcia E, Bouchard P, De Brux J, Berdah J, Frydman $R$, Schaison G, et al. Use of immunocytochemistry of progesterone and estrogen receptors for endometrial dating. J ClinEndocrinolMetab 1988;67(1):80-7.
15. Shet T, Agrawal A, Nadkarni M, Palkar M, Havaldar R, Paramar V, et al. Hormone receptors over the last 8 years in a cancer referral center in India: what was and what is? Indian J PatholMicrobiol 2009;52(2):171-4.

16. Lessey BA, Killiam AP, Metzger DA, Haney AF, Greene GL, McCarty KS. Immunohistochemical analysis of human uterine estrogen and progesterone receptors throughout the menstrual cycle. J ClinEndocrinolMetab 1988;67(2):334-40.

17. Gleeson N,Jordan M, Sheppard B, BonnarJ.Cyclicalvariation in endometrial oestrogen and progesterone receptors in women with normal menstruation and dysfunctional uterine bleeding. Eur J ObstetGynecolReprodBiol 1993;48(3):20714.

18. Wells CA, Sloane JP, Coleman D, Munt C, Amendoeira I, Apostolikas N, et al. Consistency of staining and reporting of oestrogen receptor immunocytochemistry within the Europian Union: an inter-laboratory study. Virchows Arch 2004;445(2):119-28.

19. Margarit L, Taylor A, Roberts MH, Hopkins L, Davies $\mathrm{C}$, Brenton AG, et al. MUC1 as a descriminator between endometrium from fertile and infertile patients with PCOS and endometriosis. J ClinEndocrinolMetab 2010;95(12):5320-9.

20. Papanikalaou EG, Bourgain C, Kolibianakis E, Tournaye $\mathrm{H}$, Devroey P. Steroid receptor expression in late follicular phase endometrium in GnRH antagonist IVF cycles is already altered, indicating of early luteal phase transformation in the absence of secretory changes. Hum Reprod 2005;20(6):1541-7.

*Corresponding author:

Dr Sanjay Kaushik, Shri Guru Ram Rai Institute of Medical \& Health Sciences, Dehradun, India

Phone: +91 01352522110

Email: sanjaykaushik038@gmail.com

Date of Submission : 26.02.2017

Date of Acceptance : 21.08.2017

Financial or other Competing Interests: None.
Date of Publication : 11.12.2017 